\title{
THE UNIVERSITY OF

\section{Noise2Self4Astro}

\section{Blind Denoising by Self-Supervised Neural Networks in Astronomical Datasets Yunchong Zhang, The University of Chicago \\ LSST Data Science Program @ Fermilab \\ FERMILAB-POSTER-19-109-CD}

\section{The Noise2Self Algorithm}

The foundation of the project is the noise2self algorithm. As opposed to "noise2noise" and "noise2truth", it allows us to denoise high-dimensional measurements without any prior on the signal, any estimate of the noise, or any clean training data.
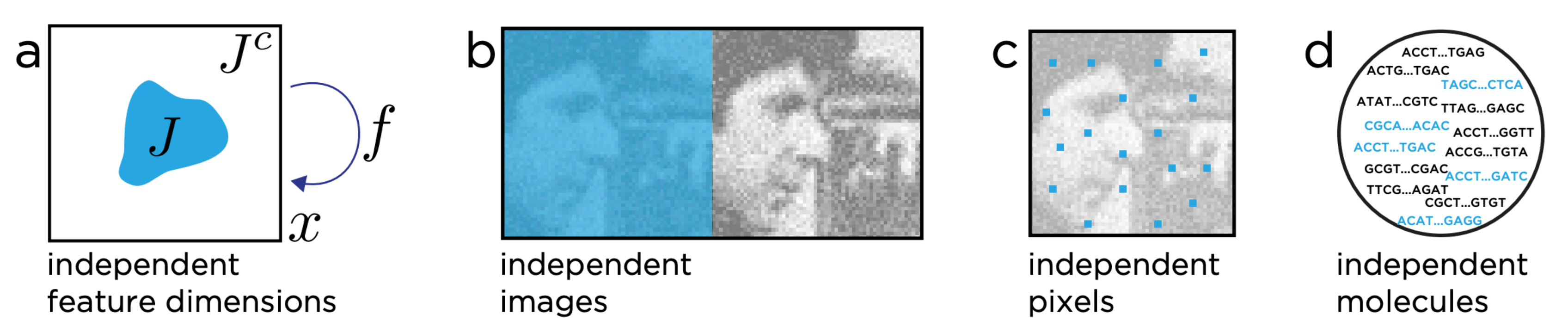

\section{Background \& Goal}

In astronomy datasets, one often acquires many images of the same part of the sky, with different shot, read-out, cosmic ray, and detector noise. Traditionally, in order to gain denoised model for astronomical objects, researchers take the average of multiple images of the same object or apply pre-processing filters such as Gaussian filter or Median filter, feeding them into "Galfit", which is a software for astronomical object model fitting.

Instead of that, we may train a network to predict one noisy image from the other based on the Noise2Self algorithm. The resulting output will be a semi-denoised image, which would later be put into galfit to complete the denoising process
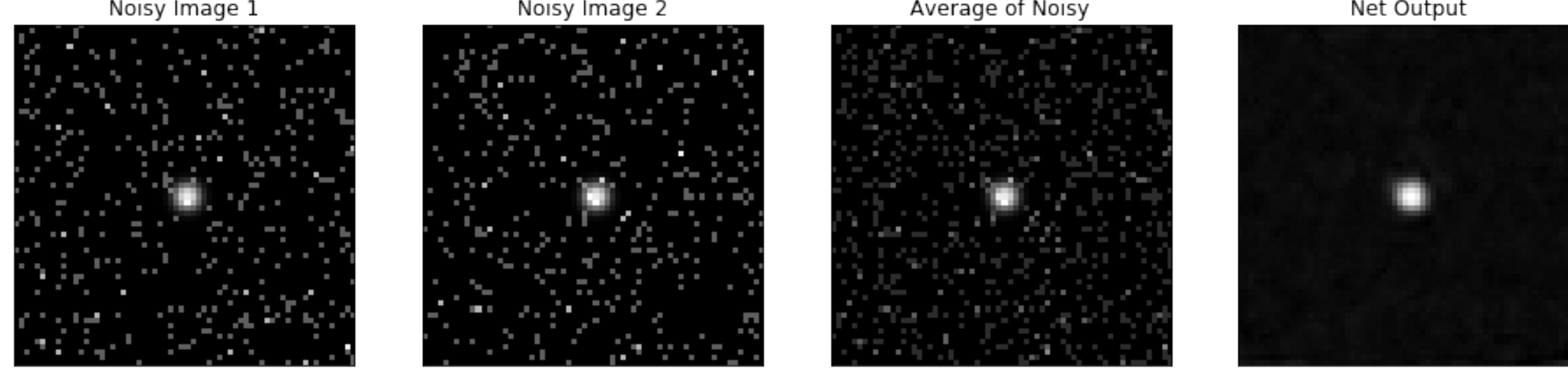

\section{Current Status}

For our initial test, we used a short UNet with two levels of upand down- sampling, with the loss function to be a mean squared error function that takes in the difference between the net output and the second noisy image. The neural net is run on the dataset of 998 sets of $128^{*} 128$ pixels images and each set of image contains the same astronomical objects under different level of noise. All of the images for the initial test are generated through injecting sersic profiles into CCD sky cutouts, as we decided to evaluate its performance based on controllable datas before we move on to real sky images.
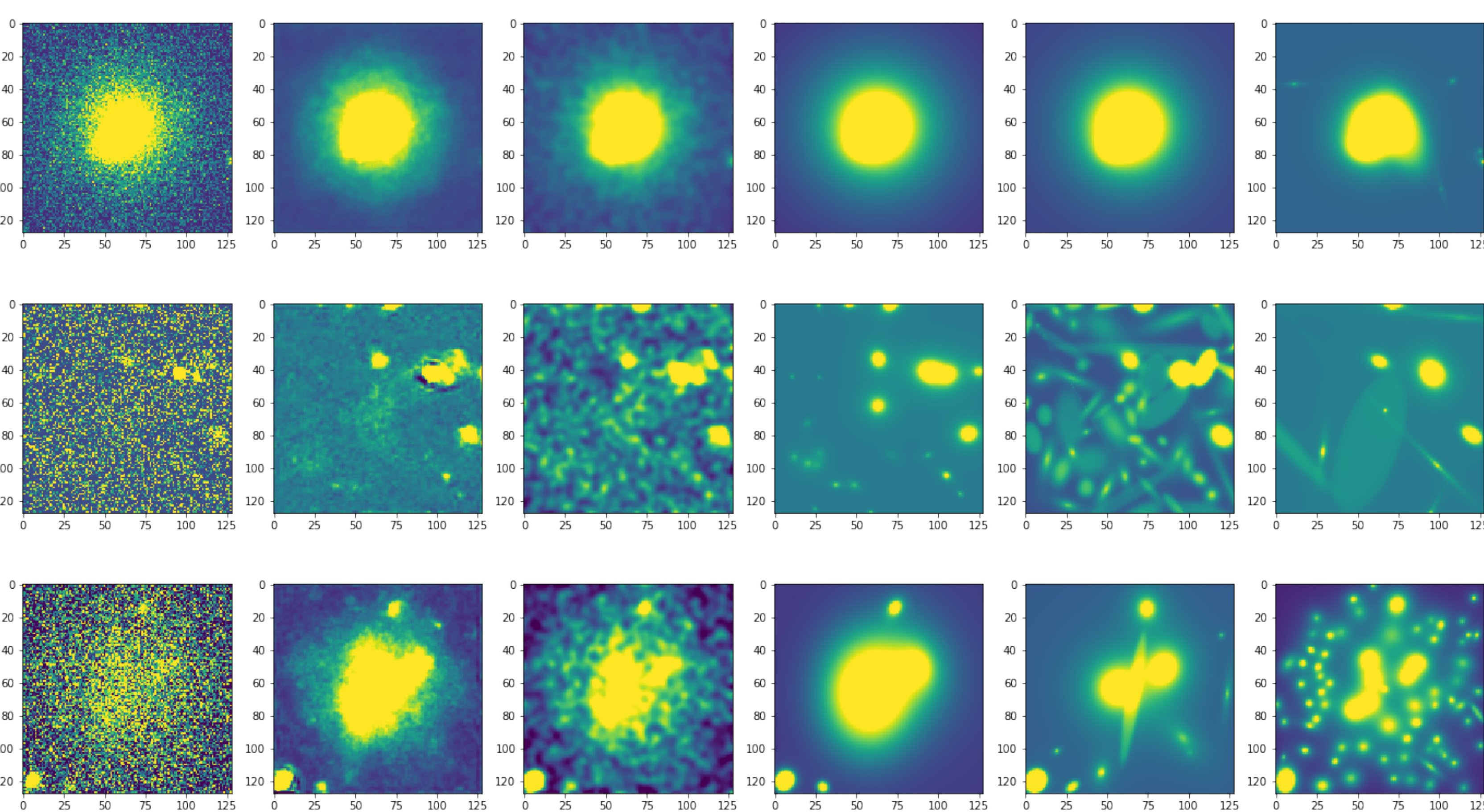

\section{Performance Evaluation}

: Denoising Methods

: Direct outputs or indirect outputs

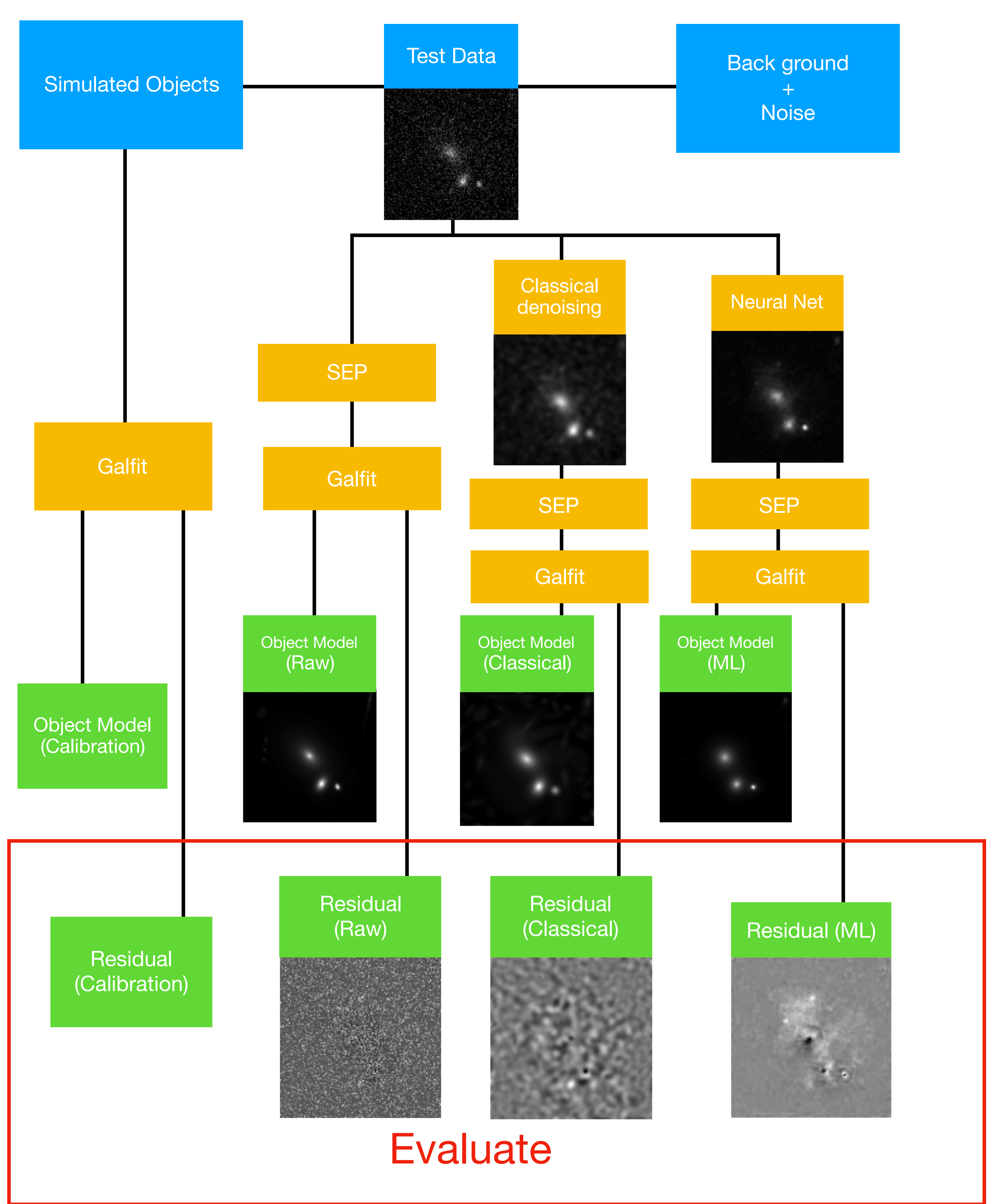

\section{Conclusion \& Future Work}

- In the initial test, our neural network has successfully removed the majority of noise in most test images and maintained crucial structure of the objects. However, it has also introduced certain extra textures, which are suspected be the cause of galfit's failure in some cases.

- In the next step of the project, we are planning to apply neural networks with more complicated architecture as well as multiple channel inputs. And in order to evaluate the residuals with ground truth noise, we plan to run the comparison test with datasets which contain completely simulated main objects and background objects.

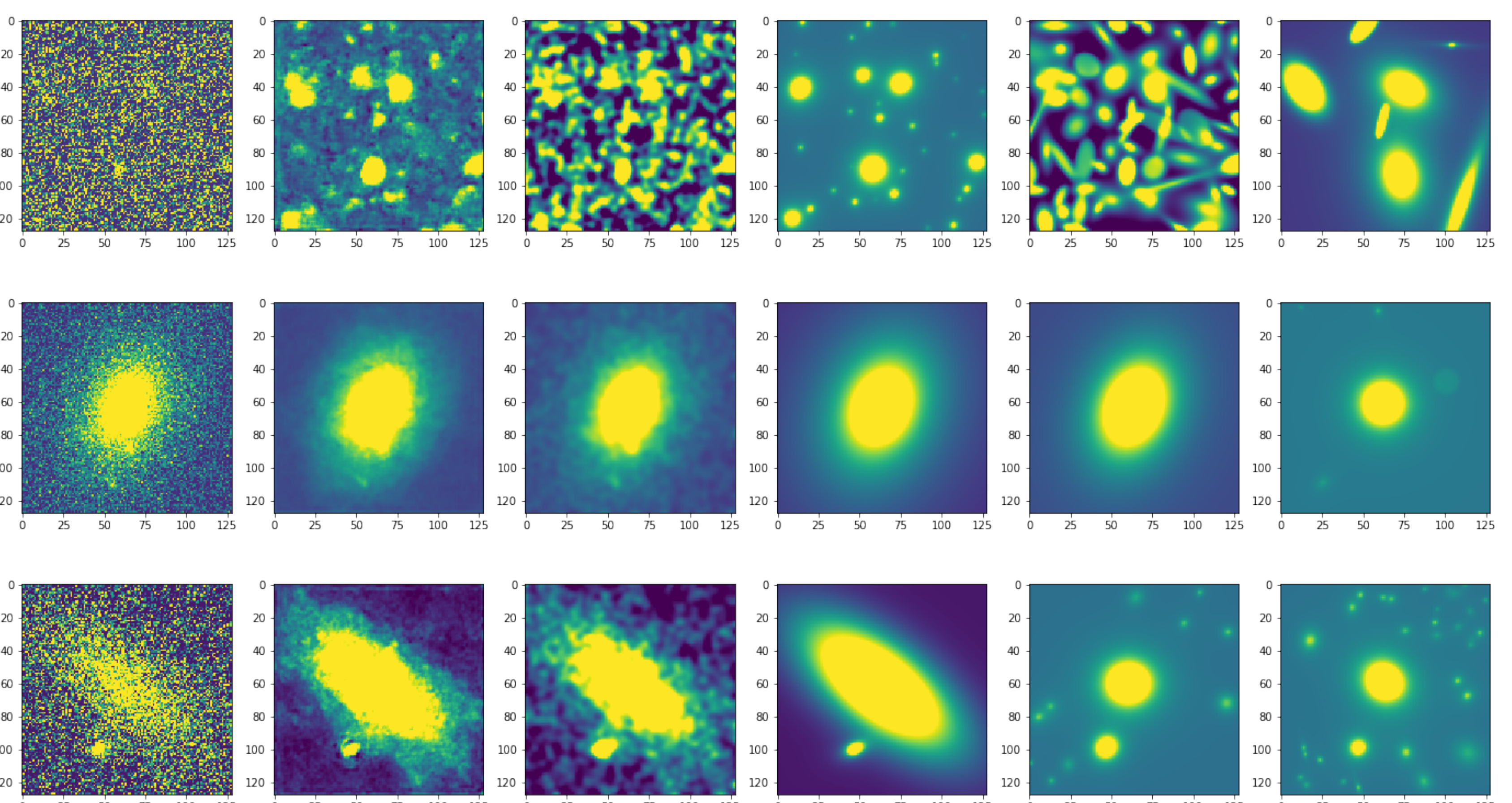

Reference: Noise2Self: Blind Denoising by Self-Supervision https://arxiv.org/abs/1901.11365v2

Acknowledgement: I would like to thank my supervisor Brian Nord for his irreplaceable support, as well as other members of the "Noise2Astro" project team for their patience and enthusiasm during my internship.

This document was prepared by Deep skies Collaboration (deepskieslab.com) using the resources of the Fermi National Accelerator Laboratory (Fermilab), a U.S. Department of Energy, Office of Science, HEP User Facility. Fermilab is managed by Fermi Research Alliance, LLC (FRA), acting under Contract No. DE-AC02-07CH11359. 